\title{
FUSION OF HIGH-RESOLUTION SATELLITE AND LIDAR DATA FOR INDIVIDUAL TREE RECOGNITION
}

\author{
Marek B. Zaremba \\ Département d'informatique et d'ingénierie \\ Université du Québec en Outaouais \\ Gatineau, QC J8Y $3 G 5$ \\ email: zaremba@uqo.ca
}

\author{
François A. Gougeon \\ Natural Resources Canada \\ Pacific Forestry Centre \\ Victoria, BC V8Z 1 M5 \\ email: fgougeon@nrcan.gc.ca
}

\begin{abstract}
A major shift in the forest inventory and management paradigm toward the use of semi-automated analysis realized on an individual tree crown basis has been made possible by recent developments in high-resolution remote sensing. This paper discusses issues related to the fusion of high-resolution satellite imagery and LIDAR (LIght Detection and Ranging) data and their application in the classification of individual trees for precision forest management. The proposed methodological approach consists in the combination of spatial filtering object detection and reconstruction methods with a rule-based Individual Tree Crown (ITC) system. Examples using QuickBird imagery combined with LIDAR data from an Alberta site (both boreal and mixed forest) demonstrate the advantages of the proposed fusion approach.
\end{abstract}

Keywords: Satellite imagery; Lidar data; Individual Tree Crown system; data fusion.

\section{Introduction}

The general trend toward precision and sustainable forestry calls for a transition from mapping relatively homogeneous forest stands and manually interpreting their content to the use of semi-automated, computer-assisted analysis of high-resolution multi-spectral images realized on an individual tree crown basis [1]. This potentially major shift in the forest inventory and management paradigm has been made possible by recent developments in high-resolution remote sensing. The high spatial and radiometric resolution of such satellites as QuickBird, OrbView3 or Ikonos facilitates visual interpretation [2,3]. Temporal resolution of image databases can be largely increased, due to the known revisit time and pointing capabilities of the satellite platform, which facilitates large-scale change-detection and monitoring of selected areas in order to keep forestry databases up-to-date. Certain analyses important for forest inventory and involving spectral change detection can be more easily performed. Maps used to estimate stratum sizes can be produced in a more automated fashion. Finally, high-resolution satellite images offer the potential for being ever more competitive in terms of price with the aerial images.
In order to obtain forest inventory information derived from volumetric characteristics, the multispectral images have to be complemented by altimetric data collected by Lidar sensors. It has been shown that Lidar can be used to estimate a wide range of forest inventory parameters, including tree heights [4], stand density [5], and canopy fuel parameters [6]. The use of Lidar data combined with digital videography for individual tree crown delineation was studied in [7].

In forest management practice, the acquisition of Lidar data is still uncommon, but the idea is gaining popularity. When acquired, it is mainly to create precise Digital Terrain Model (DTM) that are used in engineering endeavors, such as forestry road building where grade is of primary importance due the heavy loads carried by logging trucks. Getting Digital Canopy Models (DCM) to evaluate forest stand's height for wood volume is still perceived as secondary. Indeed, since forest inventories are typically done by the photo-interpretation of stereo pairs of aerial photographs, this information is readily available. However, sustainable forest management objectives and multi-resources management concerns are creating demands for more precise, accurate and timely forest inventories. We believe that most of these new requirements can realistically be met by the semi-automatic analysis of high spatial resolution satellite images done at the Individual Tree Crown (ITC) level. Such an ITC-based approach provides detailed species composition for each forest stand, as well as stands boundaries, but not the height information so important to volume assessments. Although the fusion of multispectral and LIDAR data can bring some benefits at most scales (e.g., stand heights), this paper examines the possible synergies between these two data sources at the ITC scale. Our long-term goals are the creation of individual tree inventories, with semiautomated ITC-based volume assessments and ITC-level forest management, leading towards the new era of precision forestry.

Achieving a semi-automated ITC-based interpretation of high spatial resolution digital data for forestry requires that several components work together. The key components are:

- automatic isolation of visible individual tree crowns,

- classification of individual tree crowns,

- regrouping into stands or strata,

The delineation process uses a rule-based approach to systematically follow boundaries from the inside of a specific 
crown (or cluster) to produce more distinct crowns. A nearinfrared band is typically used because of its sensitivity to illumination variations and its good response to vegetative material. That process contains numerous, context-sensitive crown separation criteria. Species classification is done on an individual crown basis as opposed to a pixel-by-pixel approach. Each crown is described by a single multidimensional vector using spectral signatures acquired for representative tree crowns of each species.

After a brief introduction to the remote sensing technology in Section 2, the ITC method is discussed in Section 3. Integration of the satellite and Lidar technology is presented in Section 4. Some results of an R\&D project that involves partners from UQO, IQAFF, Natural Resources Canada and two forest management companies from Alberta and Quebec are given.

\section{Remote Sensing Data}

The satellite imagery used in this study consists of QuickBird data acquired in the Whitecourt Forest area in north-west Alberta in August 2005. Table 1 presents the characteristics of the images.

Table 1. Spatial and spectral resolution of QuickBird images.

\begin{tabular}{|c|c|cccc|}
\hline & Panchromatic & \multicolumn{4}{|c|}{ Multispectral } \\
\hline $\begin{array}{c}\text { Spectral } \\
\text { Characteristics }\end{array}$ & Black \& White & Blue & Green & Red & $\begin{array}{c}\text { Near } \\
\text { IR }\end{array}$ \\
& 450 to $900-\mathrm{nm}$ & $\begin{array}{c}450 \text { to } \\
520-\mathrm{nm}\end{array}$ & $\begin{array}{c}520 \text { to } \\
600-\mathrm{nm}\end{array}$ & $\begin{array}{c}630 \text { to } \\
690-\mathrm{nm}\end{array}$ & $\begin{array}{c}760 \text { to } \\
900-\mathrm{nm}\end{array}$ \\
\hline Pixel Resolution & $61-\mathrm{cm}$ to $72-\mathrm{cm}$ & & 2.44 to $2.88-\mathrm{m}$ \\
\hline $\begin{array}{c}\text { Scene } \\
\text { Dimensions }\end{array}$ & $\begin{array}{c}27,552 \times 27,424 \\
\text { pixels }\end{array}$ & \multicolumn{4}{|c|}{$6,888 \times 6,856$ pixels } \\
\hline
\end{tabular}

The QuickBird images were orthorectified using Lidarderived DEM. Also the pan-sharpened version of the QuickBird imagery was available.

High-density Lidar data were acquired with an Optec 3100 system over a $57 \mathrm{~km}^{2}$ area within the Whitecourt Forest, Alberta, in the summer of 2005. The data set was defined in UTM zone 11, NAD83, orthometric heights, and was provided in the form of an ASCII text file consisting of $X Y Z$ coordinates, intensity of the Lidar returns, and class identifiers assigned to each return.

\section{Individual Tree Crown (ITC) Approach}

\subsection{Methodology}

For the typical medium density Canadian forest, most dominant and co-dominant tree crowns are visible on high spatial resolution (< $100 \mathrm{~cm} / \mathrm{pixel})$ satellite imagery and can often be separated based on the shade found between them. After having masked the non-forested areas, a first computer program (ITCVFOL) follows the valleys of shade that exist between the much brighter tree crowns in the spectral topography of a panchromatic image. A second program (ITCISOL) improves and completes the delineation process base on a set of rules aimed at the remaining tree clusters. Typically, depending on the spatial resolution of the image, 65$80 \%$ of the main visible tree crowns can be separated by these two processes. The individual tree crowns and remaining tree clusters are from then on collectively referred to as ITCs.

Following a supervised classification protocol, spectral signatures are created for all the classes of interest (i.e., tree species or tree species in special situations). That is, the multispectral values of the pixels contained within these objects (ITCs), or parts thereof (e.g., lit side of the objects), are summarized to create ITC signatures (i.e., mean multispectral vector and covariance matrix) and the signatures of designated ITCs (i.e., judged to be of the same class) are combined to create species signatures (i.e., average mean vector, covariance of the ITC means). The classification process then compares one-by-one all of the ITC's signatures with the species (classes) signatures and using a maximum likelihood decision rule assigns them a class (within a specified confidence interval). Finally, because (at this point in time) forest stands are still the preferred management unit and because Geographic Information Systems are still hard-pressed to deal with millions of objects (tree polygons), the ITCs are regrouped into forest stands based on species composition, crown closure and stem density. Forest stand polygon shapes and contents comparable, yet superior, to existing forest inventories have been achieved from IKONOS and QuickBird images.

\subsection{Crown Delineation Results}

An image of the NIR band of a sample plot used as an example illustrating the method is shown in Fig. 1a. The image depicts a road on the left side and an area of a forest of different height on the rest of the image. Large zones of shadow can be noticed, which makes the task of tree crown delineation and classification more difficult.
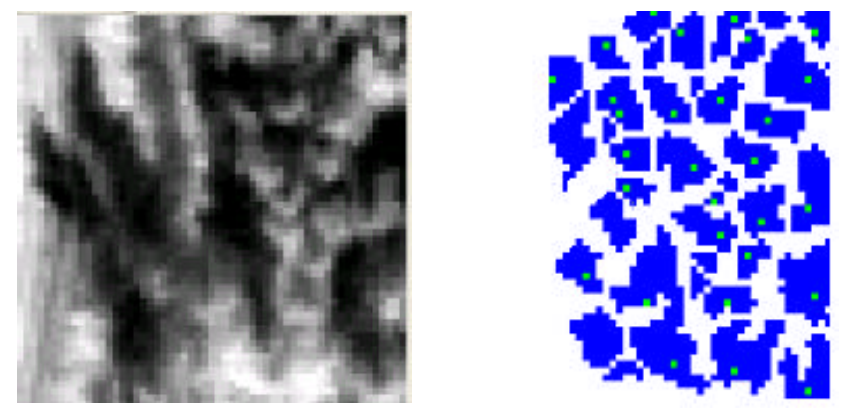

Fig. 1. NIR band of the sample plot image (a) and the ITC tree crown delineation results (b).

The results of the application of the ITC tree delineation procedure, after masking out the road area, are shown in Fig. 1b. In addition to the crown polygons, the image also includes the tree tops, shown as lighter spots in the tree crown zones. 
While the ITC procedure performs well in the dense forest areas, addition of the altimetric data can enhance the procedure especially in low-vegetation and shadow-covered zones.

\section{Satellite and Lidar Data Aggregation}

\subsection{Data Synergy}

The availability of Lidar data can facilitate and improve this ITC analysis process in many ways. One of the primary requirements of the above process is to successfully mask the non-forested areas, otherwise, trees will be found where non exist. Although non-vegetated areas can easily be separated from vegetated areas using simple thresholds comparing near infrared radiances with visible light radiances, thus removing roads and man-made structures from the ITC analysis, the separation of vegetated areas from forested areas is more demanding. Some separation can be achieved based on texture analysis, forested areas having significantly more texture than grassy or even shrubbery areas, at least for the spatial resolutions under consideration here. This works well for relatively large homogeneous areas, but texture being an areabased measurement, area boundaries will rarely be precise. Moreover, it typically failed for open forests, where trees and understorey shrubs are seen side by side. The availability of co-registered Lidar data reduces this separation exercise to the use of a simple height threshold. Additionally, this can be achieved with high or low point density Lidar data, with corresponding spatial accur acy tradeoffs.

With high point density Lidar data $\left(\sim 5\right.$ points $\left./ \mathrm{m}^{2}\right)$, a precise digital canopy model (DCM) can be achieved by subtracting the digital terrain model (DTM) from the digital elevation model (DEM). This is accomplished by subtracting a surface generated from the last Lidar returns (assumed ground) from one generated from the first returns (assumed vegetation above ground). The resulting DCM looks and functions like a panchromatic image on which the crown delineation software can be run. This produces a slightly different delineation of crowns from that of the QuickBird image. In the later, clusters of trees are typically encountered when trees line-up at $90^{\circ}$ to the sun illumination because of the lack of shade between them. In the former, these crowns may become separated. Conversely, crown separation in the DCM encounters difficulties (i.e., creates tree clusters) at the larger acquisition angles, as returns from the ground or understorey are less likely. Additionally, some image-generated tree crowns are often inappropriately delineated due to the fact that illuminated understorey material got linked with them.

A synergic effect is achieved by using both media in the crown delineation process [8]. This is accomplished by running the valley following program (ITCVFOL) on each media and combining the resulting bitmaps (an OR operator) before applying the rule-based program (ITCISOL). This results in more accurate crown delineations and in more individual tree crowns being separated.

Synergy between Lidar and QuickBird data also occurs on other levels. For example, the Lidar-generated DTM benefits the ITC classification. Indeed, the radiances of a given forest species on south-facing slopes can be quite different from that of the same species facing north (i.e., due to illumination factors). They have to be treated as two distinct classes and the availability of slope and aspect information strengthen the classification of these classes. And of course, the ultimate contribution of Lidar data is towards ITC-based volume assessments. When combined with delineated tree crowns, the Lidar-generated DCM or preferably, the original Lidar first returns dataset, makes individual tree height available for the calculation of tree volumes as a function of crown area and height.

\subsection{Spatial Filtering of Lidar Data}

Running the tree top detection and crown delineation procedure directly on the Lidar-generated DCM presents a number of difficulties. Because of a rugged shape of the canopy surface, the results tend to generate more than one treetop point for each tree crown. The points are situated too close to each other. Imposing a minimum crown size offers a partial solution. However, this solution will not take into account the shape of the tree crown. The proposed solution is based on a modified multi-scale image relevance function (IRF) approach [9], [10], and consists of three stages: detection of the tree crown, detection of the crown center, and the verification of the resulting crown. Since the Lidar data offer essentially the geometrical information, the comparison with the ITC approach will be limited to the stage of crown delineation.

\section{a) Detection (delineation) of the tree}

Multi-scale image relevance function (IRF) is computed within the given scale range $\left(S_{\max }, S_{\min }\right)$ in point $(i, j)$ according to a formula that includes terms related to the intensity, contrast (height), homogeneity, size and shape symmetry of the object.

$$
\begin{aligned}
& \Phi\left[f(i, j), S_{m}\right]=c\left(i, j, S_{m}\right)-\alpha \cdot d\left(i, j, S_{m}\right) \\
& +\beta \cdot s\left(i, j, S_{m}\right)+\gamma \cdot p\left(i, j, S_{m}\right)
\end{aligned}
$$

where $f(i, j)$ is the input image, $c\left(i, j, S_{m}\right)$ is an estimate of the local contrast in point $(i, j), d\left(i, j, S_{m}\right)$ is an estimate of the mean intensity deviation in the object region used to measure its homogeneity, $s\left(i, j, S_{m}\right)$ is the difference between the current scale and the minimal possible scale, and $p\left(i, j, S_{m}\right)$ is the estimate of the shape symmetry (compactness). Coefficients $\alpha$, $\beta$ and $\gamma$ are constraint which control the contribution of the last three terms in Eq. (1) relatively to the local contrast.

\section{b) Detection of the centre}

The positions of local maxima of the non-linear matched filter (1) indicate on the locations of tree tops:

$$
\left(i_{f}, j_{f}\right)_{l}=\arg \max _{(i, j) \in A} \max _{k}\left\{\Phi\left[\left(f(i, j), S_{k}\right],(i, j) \notin \Gamma_{l}\right\},\right.
$$

where $A$ is a region of interest, and $\Gamma_{l}$ is the masking region. 


\section{c) Object verification}

This step compares estimated object features $x_{1}, \ldots, x_{N}$ in the current maximum point $\left(i_{f} j_{f}\right)_{l}$ with corresponding thresholds ? $\ldots, ?_{N}$, i.e., if $x_{1}>?_{1}$ and,$\ldots$, and $x_{N}>?_{N}$, then point $\left(i_{f} j_{f}\right)_{l}$ is the tree crown top.

The results of the application of the above procedure on the Lidar-generated DCM (Fig. 2a) are shown in Fig. 2b. Only high-vegetation trees are detected. The number of trees can be controlled by the value of $\beta$ in (1).

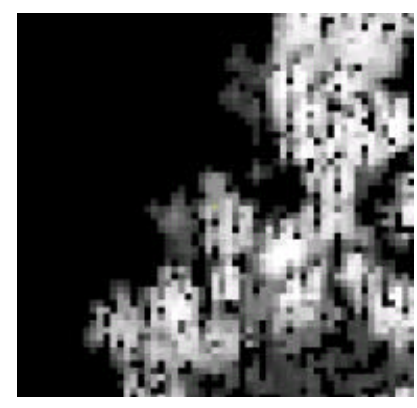

(a)

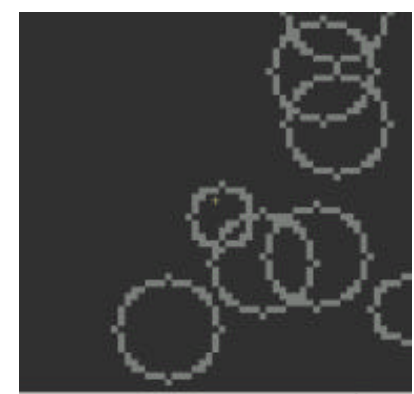

(b)
Fig.2. Digital Canopy Model (a) and the scales of the detected tree crowns (b).

The results of the tree detection can be compared with the DEM surface shown in Fig. 3.

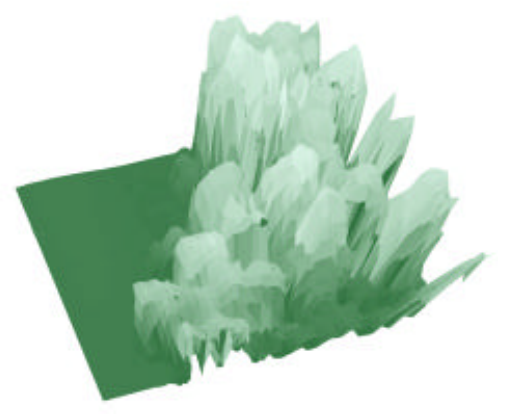

Fig. 3. Lidar-derived DEM surface.

Extension of the fusion of multi-source data to the classification process can be readily obtained by projecting the original high dimensional data onto a low dimensional space, where all the classes are well separated. Application of an optimal linear dimensionality reduction method, such as Fisher linear discriminant analysis, which maximizes the ratio of between-class scatter matrix determinant to within-class scatter matrix determinant, will generate a surface used as $f(i, j)$ in (1).

\section{Conclusions}

Synergy between high-resolution satellite imagery and data sets from altimeter sensors at the ITC scale was examined. The use of a spatial filtering procedure, which operates on the Lidar data, and the results of which guide the ITC-based tree crown delineation and classification process, was proposed. The multi-scale spatial filtering includes four terms related to the intensity, contrast (height), homogeneity, size and shape symmetry of the object. The parameters of the filter can be self-tuned, allowing for the automation of the tree detection process. Further research is carried out on the fusion of both techniques for the purpose of tree crown classification.

\section{Acknowledgements}

Funding by the NSERC Collaborative Research and Development program (Grant CRDPJ 311302) is gratefully acknowledged.

\section{References}

[1] F.A. Gougeon and D.G. Leckie, "Forest information extraction from high spatial resolution images using an individual tree crown approach," NRC CFS Pacific Forestry Centre Rep. BC-X-396, Victoria, B.C., 2003.

[2] P. Holmgren, T. Thuresson, "Satellite remote sensing for forestry planning: A review," Scandinavian Journal of Forest Research, vol. 13, pp. 90-110, 1998.

[3] J.V. Johnson, P. Greenfield, and J. Ellenwood, "Using Ikonos satellite imagery for forest pest mapping," Proc. Forest Service Remote Sensing Conference, San Diego, USA, 2002.

[4] S. Magnussen, P. Eggermont, and V.N. LaRiccia, "Recovering tree heights from airborne laser scanner data," Forest Science, vol.45, no.3, pp. 407-422, 1999.

[5] J. Means, S. Acker, B. Fitt, M. Renslow, L. Emerson, and C. Hendrix, "Predicting forest stand characteristics with airborne scanning Lidar," Photogrammetric Engineering and Remote Sensing, vol. 66, pp. 1367-1371, 2000.

[6] H.-E. Andersen, R.J. McGaughey, and S.E. Reutebuch, "Estimating forest canopy fuel parameters using LIDAR data," Remote Sensing of Environment, vol. 94, pp. 441449, 2005.

[7] F.A. Gougeon, B.A. St-Onge, M. Wulder, and D.G. Leckie, "Synergy of airborne laser altimetry and digital videography forindividual tree crown delineation," Proc. $23^{\text {rd }}$ Canadian Symposium on Remote Sensing (CD-ROM), Sainte-Foy, QC, Canada, 2001.

[8] D.G. Leckie et al., "Combined high density lidar and multispectral imagery for individual tree crown analysis," Can. J. of Remote Sensing . vol. 29, no. 5, pp. 1-17, 2003.

[9] R.M. Palenichka, P. Zinterhof, M. Volgin, 'Detection of local objects in images with textured background by using multi-scale relevance function," Proc. SPIE Int. Annual Symp., San Diego, USA, vol. 4121, pp. 158-170, 2000.

[10]M.B. Zaremba, R.M. Palenichka, "Relevance-based Content Modeling and Object Retrieval from Multi-source Image Data," Concurrent Engineering: Research and Applications, vol. 13, no. 2, pp. 155-166, 2005. 